\title{
Intrinsic Non-Radiative Voltage Losses in Fullerene-Based Organic Solar Cells
}

Johannes Benduhn ${ }^{1, *}$, Kristofer Tvingstedt2,* , Fortunato Piersimoni ${ }^{3}$, Sascha Ullbrich ${ }^{1}$, Yeli Fan ${ }^{4, \ddagger}$, Manuel Tropiano ${ }^{5}$, Kathryn A. McGarry ${ }^{6}$, Olaf Zeika ${ }^{1}$, Moritz K. Riede ${ }^{7}$, Christopher J. Douglas ${ }^{6}$, Stephen Barlow ${ }^{4}$, Seth R. Marder ${ }^{4}$, Dieter Neher $^{3}$, Donato Spoltore ${ }^{1}$, Koen Vandewal ${ }^{1, *}$

*Corresponding authors (johannes.benduhn@iapp.de, ktvingstedt@physik.uni-wuerzburg.de, koen.vandewal@iapp.de)

${ }^{1}$ Dresden Integrated Center for Applied Physics and Photonic Materials (IAPP) and Institute for Applied Physics, Technische Universität Dresden, Nöthnitzer Str. 61, 01187 Dresden, Germany

2 Experimental Physics VI, Julius-Maximilian University of Würzburg, 97074 Würzburg, Germany

${ }^{3}$ Institute of Physics and Astronomy, University of Potsdam, Karl-Liebknecht-Str. 24-25, 14476 Potsdam, Germany

${ }^{4}$ Center for Organic Photonics and Electronics and School of Chemistry and Biochemistry, Georgia Institute of Technology, Atlanta, Georgia 30332-0400, United States

${ }^{5}$ Chemistry Research Laboratory, University of Oxford, Mansfield Road, Oxford OX1 3TA, United Kingdom

${ }^{6}$ Department of Chemistry, University of Minnesota, 207 Pleasant St SE, Minneapolis, 55455, United States

7 Department of Physics, University of Oxford, Parks Road, Oxford OX1 3PU, United Kingdom

$\ddagger$ Current address: School of Chemistry and Chemical Engineering, Southeast University, Nanjing 211189, Jiangsu, P.R. China

\section{Abstract}

Organic solar cells demonstrate external quantum efficiencies and fill factors approaching those of conventional photovoltaic technologies. However, as compared to the optical gap of the absorber materials, their open-circuit voltage is much lower, largely due to the presence of significant nonradiative recombination. In this work, we study a large data set of published and new material combinations and find that non-radiative voltage losses decrease with increasing charge-transfer state energies. This observation is explained by considering non-radiative charge-transfer state decay as electron transfer in the Marcus inverted regime, being facilitated by a common skeletal molecular vibrational mode. Our results suggest an intrinsic link between non-radiative voltage losses and electron-vibration coupling, indicating that these losses are unavoidable. Accordingly, the theoretical upper limit for the power conversion efficiency of single junction organic solar cells would be reduced to about $25.5 \%$ and the optimal optical gap increases to $(1.45-1.65) \mathrm{eV}$, i.e. $(0.2-0.3) \mathrm{eV}$ higher than for technologies with minimized non-radiative voltage losses. 
The highest internal quantum efficiencies $\left(I Q E_{\mathrm{PV}}\right)$ reported for organic solar cells (OSCs) approach $100 \%{ }^{1}$. External quantum efficiencies $\left(E Q E_{\mathrm{PV}}\right)$ can reach over $85 \%^{2}$, while fill factors $(F F)$ of $80 \%$ have been demonstrated ${ }^{3}$. However, the current highest power conversion efficiency $(P C E)$ is $11.7 \%{ }^{4}$, mainly limited by the low open-circuit voltage $\left(V_{\mathrm{OC}}\right)$ of these materials. Indeed, as compared to the optical gap of the absorber material, $V_{\mathrm{OC}}$ in OSCs is much lower than for other technologies such as crystalline silicon, GaAs, or perovskite solar cells $(\mathrm{SCs})^{5,6}$, for reasons not yet understood.

In an idealized SC, the highest $V_{\mathrm{OC}}$ is reached when recombination can only occur radiatively, from the lowest energy excited state to the ground state. As these transitions are directly related to the inverse process of absorption, the presence of radiative voltage losses is inevitable ${ }^{7}$. However, there is always significant non-radiative recombination taking place simultaneously, reducing $V_{\text {oc. }}$ Indeed, the discovery and removal of non-radiative surface recombination was the breakthrough that resulted in a high $V_{\mathrm{OC}}$ for $\mathrm{GaAs}^{8}$. In OSCs, currently, voltage losses due to non-radiative recombination are much higher than in other technologies ${ }^{5,9,10}$.

A complete picture of the total voltage losses in OSCs does not only consider recombination losses but also electron-transfer losses due to the conversion of strongly bound excitons in the neat donor (D) or acceptor (A) material into more delocalized charge-transfer (CT) states. It has recently been shown that it is possible to create systems with very low electron transfer losses $(<50 \mathrm{meV})$, while maintaining a high free-carrier generation yield $11,12,13,14$. Voltage losses due to radiative CT state recombination, $\Delta V_{r}$, add up to approximately $250 \mathrm{mV}$ and can be reduced by lessening the D:A interface area ${ }^{15}$. This leaves non-radiative recombination as the currently least well understood process in OSCs, but responsible for a significant fraction of the voltage losses. More information on the quantities of voltages losses can be found in Supplementary Note 1. Non-radiative voltage losses, $\Delta V_{\mathrm{nr}}$, are defined as ${ }^{16}$ :

$$
\Delta V_{\mathrm{nr}}=\frac{k_{\mathrm{B}} T}{q} \ln \left(\frac{1}{E Q E_{\mathrm{EL}}}\right)
$$

The quantum yield of the electroluminescent $(E L)$ emission $\left(E Q E_{\mathrm{EL}}\right)$ can be measured when driving the SC with forward injection current and equals unity if non-radiative recombination is absent, making $\Delta V_{\mathrm{nr}}=0$ in that case. However, $\Delta V_{\mathrm{nr}}$ amounts typically to about $350 \mathrm{mV}$, which corresponds to an $E Q E_{\mathrm{EL}}$ of $\sim 10^{-65,9,6,17}$. As compared to the electron transfer losses and the radiative losses, the non-radiative voltage losses therefore form a significant fraction of the total voltage losses in OSCs. 
In this work, we study a large set of D-fullerene material systems, comprising vacuum processed small molecules (SM) and solution processed polymers and small molecules, implemented into both bulkheterojunction $(\mathrm{BHJ})$ and planar heterojunction $(\mathrm{PHJ})$ device architectures. For these, in total more than 100 different, D-A combinations, the energy difference between the ground state and CT state (i.e. $E_{\mathrm{CT}}$ ) spans $1 \mathrm{eV}$, ranging from $0.7 \mathrm{eV}$ to $1.7 \mathrm{eV}$. We observe a clear correlation of $E Q E_{\mathrm{EL}}$ with $E_{\mathrm{CT}}$, such that the resulting $\Delta V_{\mathrm{nr}}$ increases with decreasing $E_{\mathrm{CT}}$. We suggest this striking correlation to be associated with an increased wave-function overlap between the relaxed CT state and higher order vibrational modes of the ground state, in analogy with the "energy-gap law" (EGL) for non-radiative decay in large organic molecules and their complexes ${ }^{18,19,20,21,22,23}$. We find no change in $\Delta V_{\mathrm{nr}}$ upon replacement of carbon-hydrogen bonds by carbon-deuterium bonds, excluding $\mathrm{C}-\mathrm{H}$ vibrations as the dominant vibrational mode. Analysis of the low energy tail of CT emission spectra points to skeletal carbon-carbon bonds as the main mediator for the non-radiative decay of $D-C_{60} C T$ states. This type of non-radiative recombination would therefore be intrinsic to organic materials and accordingly conceptually different from the usually discussed origins of non-radiative recombination in other SC technologies such as defects, surface recombination, or Auger recombination ${ }^{8}$. Taking into account these energy-gap dependent non-radiative voltage losses, the theoretically accessible maximum PCE for OSCs is reduced to about $25.5 \%$, being $25 \%$ lower as compared to the Shockley-Queisser (SQ) $\operatorname{limit}^{24}$. Furthermore, the optimal optical gap for organic absorber molecules used in single junction OSCs would range between $1.45 \mathrm{eV}$ and $1.65 \mathrm{eV}$, blue-shifted by (0.2-0.3) eV as compared to the optimal bandgap of an ideal SCs without non-radiative voltage losses.

\section{Voltage Losses Increase with Decreasing Energy of CT State}

In order to span a needed wide range of $E_{\mathrm{CT}}$ values, we used a variety of $\mathrm{D}$ molecules mixed with fullerenes, of which the chemical names and architectures are listed in Supplementary Tables 3, 4, and 5. To ensure the generality of our study, we analysed a very large set of both vacuum processed small molecules (SM) (see Supplementary Tables 2 and 3) as well as solution processed polymers in both $\mathrm{BHJ}$ and $\mathrm{PHJ}$ device architectures (see Supplementary Table 4). Additionally, we included recently published OSCs for which $E Q E_{E L}$ data was available (see Supplementary Table 5). For each D-fullerene based device, accurate determination of $E_{\mathrm{CT}}$ is conducted by a Gaussian fit of the low energy tail of the 
CT band in the EQE $\mathrm{PV}$ spectra, following the method outlined earlier ${ }^{9}$ (see Supplementary Tables 3, 4, and 5). Figure $1 \mathrm{a}$ shows that the $V_{\mathrm{OC}}$ of these devices, measured at standard testing conditions (i.e. $1000 \mathrm{~W} / \mathrm{m}^{2}$ ), clearly correlates with $E_{\mathrm{CT}}$. The offset always amounts to more than $0.5 \mathrm{eV}$. In the radiative SQ limit, the voltage losses would be smaller than $0.3 \mathrm{eV}$ with a slope of $V_{\mathrm{OC}}\left(E_{\mathrm{CT}}\right)$ slightly below unity ${ }^{24}$. However, our extensive data set shows a slope of $V_{\mathrm{OC}}\left(E_{\mathrm{CT}}\right)$ that is higher than unity, indicating that the overall voltage losses $\left(\Delta V_{O C}=E_{C T} / q-V_{O C}\right)$, due to recombination of charge carriers, in fact decrease when $E_{C T}$ increases.

\section{Figure 1 (placeholder)}

To accurately determine the voltage losses, exclusively due to non-radiative recombination, we employ two independent but complementary methods. For high $E_{\mathrm{CT}}$ materials, a measurement of the $E Q E_{\mathrm{EL}}$ and the use of Equation (1) yields $\Delta V_{\mathrm{nr}}$. For the lower $E_{\mathrm{CT}}$ materials such a measurement is difficult due to the spectral range of CT emission being deeper in the infra-red and the considerably lower values of $E Q E_{\mathrm{EL}}$. For these $\mathrm{D}: \mathrm{C}_{60}$ blends, a second method for determining $\Delta V_{\mathrm{nr}}$ involves the calculation of the maximum value of $V_{\mathrm{OC}}$, under the assumption that non-radiative recombination would be absent ( $\left.V_{\mathrm{OC}, \mathrm{rad}}\right)$, using sensitively measured $E Q E_{\mathrm{PV}}$ spectra ${ }^{16,25}$. This yields $\Delta V_{\mathrm{nr}}$ as the difference between the calculated $V_{\mathrm{OC}, \text { rad }}$ and the measured $V_{\mathrm{OC}}$.

In Figure 1b, $\Delta V_{\mathrm{nr}}$ values obtained via both methods coincide well when measured on the same device and are plotted as a function of $E_{\mathrm{CT}}$. They are indicated by open and filled circles, respectively. A trend is clearly visible, with $\Delta V_{\mathrm{nr}}$ increasing more than $300 \mathrm{mV}$ when $E_{\mathrm{CT}}$ decreases from $1.7 \mathrm{eV}$ to $0.7 \mathrm{eV}$.

\section{Energy-Gap Law for Non-Radiative Recombination}

The observed trend in Figure $1 \mathrm{~b}$ suggests that a common mechanism is responsible for the high $\Delta V_{\mathrm{nr}}$ in all OSCs. To study this in detail, we focus on a subset of samples shown in Figure 1 by black circles. These devices comprise 24 different $D$ molecules co-evaporated with $C_{60}$ as electron acceptor at a constant donor molar ratio of $6 \%$. The full chemical names and structures are listed in Supplementary Table 2. The low molar content of the $D$ molecules ensures that $C_{60}$ is the main absorber, yielding a comparable photo-generation rate for all photovoltaic devices. Crucially, the use of diluted Ds avoids a substantial influence of morphological effects on the energy levels and recombination rates, originating from various degrees of molecular ordering $26,27,28$, and different D-A interface areas ${ }^{15}$. Moreover, these 
devices are vacuum processed using materials which allow a significant purification via vacuum sublimation. The contacts and transport layers are also identical for this subset of devices. In Figure 1b, the low-donor-content devices are among those with the lowest $\Delta V_{\mathrm{nr}}$, indicating a more optimized $V_{\mathrm{OC}}$, and show indeed a significantly less scattered trend than the other devices. For Ds with higher $E_{\mathrm{CT}}$ and consequently higher $V_{\mathrm{OC}}$, the chosen low-donor-content devices yield respectable PCEs, mainly limited by the weak absorption of $\mathrm{C}_{60}{ }^{29}$. Further information on the $j \mathrm{~V}$-characteristics of these OSCs is listed in Supplementary Table 3.

Intrinsic non-radiative recombination in large organic molecules and their complexes has been classically described by the $E G L^{30,18,31}$. In analogy, we consider non-radiative recombination at $D: C_{60}$ interfaces as an electron transfer in the inverted Marcus regime (Figure 2), being characterized by a decreased transition rate when the free energy of the process is increased ${ }^{32}$. Indeed, the non-radiative electron transfer rate from the lowest vibrational energy CT state to the iso-energetic vibrational ground state is proportional to the vibrational wave-function overlap between these two states which decreases with an increasing free energy difference, $E_{\mathrm{CT}}$, as shown for our data in Figure $1 \mathrm{~b}$.

\section{Figure 2 (placeholder)}

The high frequency modes $\left(h v_{v} \geq k_{\mathrm{B}} T\right)$, depicted in Figure 2, describe skeletal vibrations on an atomic level, i.e. they correspond to low mass vibrations ${ }^{33}$. In literature, these vibrations are assigned to either hydrogen-carbon bonds or types of carbon-carbon bonds ${ }^{18,31}$. For simplicity, all high frequency modes are treated with a mean frequency $\bar{v}_{v}{ }^{30}$. Due to their high energy, these vibrational modes have to be treated quantum mechanically with distinct energy levels, as indicated in Figure 2 with $j=\{0 \ldots n\}^{18}$. Additionally in organic molecules, there are low frequency modes $\left(h v_{\mathrm{S}} \ll k_{\mathrm{B}} T\right.$ ) attributed to the deformation of molecules upon charging, resulting in structural relaxation quantified by a relaxation energy $\lambda_{\mathrm{s}}$. Those classical modes are responsible for the line shape broadening of the sub-gap absorption of the CT state ${ }^{34}$. Taking both high and low frequency vibrational modes into account, the non-radiative transition rate $k_{\mathrm{nr}}$ can be described as being proportional to the square of the electronic coupling between CT state and ground state $(V)$ and the Franck-Condon $(F C)$ factor, which is the sum of all wave-function overlaps and assumes the Born-Oppenheimer approximation ${ }^{30,18}$ :

$$
k_{\mathrm{nr}} \propto V^{2} \cdot F C\left(g=E_{\mathrm{CT}}\right) \text { with }
$$




$$
F C(g)=\left(4 \pi \lambda_{\mathrm{S}} k_{\mathrm{B}} T\right)^{-1 / 2} \sum_{j=0}^{\infty} \frac{e^{-S_{S}}}{j !} \cdot \exp \left[-\frac{\left(g-j h \bar{\nu}_{v}-\lambda_{\mathrm{s}}\right)^{2}}{4 \lambda_{\mathrm{s}} k_{\mathrm{B}} T}\right]
$$

Where $\frac{\exp (-S) S^{j}}{j !}$ describes the wave-function overlap of the high frequency modes between the lowest vibrational CT state $(i=0)$ and ground state with quantum number $j$, and $S$ represents the ratio of the high-frequency relaxation energy and the mean phonon-energy $S=\frac{\lambda_{v}}{h \bar{\nu}_{v}}$.

The radiative recombination of the CT state, with the emission rate constant $k_{\mathrm{r}}$ is, similar to the nonradiative rate, proportional to $V^{2}$, and a $F C$ factor ${ }^{18}$, as schematically indicated in Figure $2 \mathrm{a}$ :

$$
\frac{k_{\mathrm{r}}(v)}{v} \propto V^{2} \Delta \mu^{2} \cdot F C\left(g=E_{\mathrm{CT}}-h v\right),
$$

where $\Delta \mu$ is the difference of the dipole moment of the initial and the final state. Using Equation (1)-(4) and the assumption that $\Delta \mu$ and $\lambda_{\nu}$ do not vary significantly for the studied material blends, we derive the following approximation for $\Delta V_{\mathrm{nr}}$, depending on $E_{\mathrm{CT}}, \lambda_{\mathrm{s}}$, and $h \bar{v}_{v}$. Further details about the approximations are provided in Supplementary Note 2.

$$
\Delta V_{\mathrm{nr}} \propto A-B \frac{E_{\mathrm{CT}}-\lambda_{\mathrm{s}}}{h \bar{\nu}_{v}}
$$

Note that, due to the fact that both $k_{\mathrm{r}}$ and $k_{\mathrm{nr}}$ are proportional to $\mathrm{V}^{2}$, which depends on the D-A combination, $E Q E_{\mathrm{EL}}$ and $\Delta V_{\mathrm{nr}}$ do not depend on $V^{2}$. As shown in Figure 3a, this simplified formula describes the experiments qualitatively well, with $A=(574 \pm 27) \mathrm{mV}$ and $B^{\prime}=\frac{B}{h \bar{v}_{v}}=(184 \pm 26) \mathrm{mV} / \mathrm{eV}$, and indicates that non-radiative voltage losses are especially high for D-A combinations with low $E_{\mathrm{CT}}$. Note that there is significant scatter in the overall data, possibly due to deviations of $\Delta \mu$ and $\lambda_{v}$ within the studied blends, or that there are additional loss mechanisms which go beyond the intrinsic ones described by the EGL.

\section{Non-Radiative Recombination and Low Energy Emission}

We highlight that Equation (2) and Equation (4) imply that both the radiative and non-radiative recombination rate depend on the wave-function overlap between the lowest vibrational CT state and the vibrational level of the ground state. The emission spectrum as a function of $E_{\mathrm{CT}}-\lambda_{\mathrm{s}}-h v$ is, therefore, predicted to have a similar slope as the $E Q E_{\mathrm{EL}}$ as a function of $E_{\mathrm{CT}}-\lambda_{\mathrm{s}}$. In Figure $3 a$, this 
comparison is exemplarily illustrated for the CT emission of four $D: C_{60}$ combinations, indeed showing a fairly good agreement, indicating that the applied theory is consistent.

\section{Figure 3 (placeholder)}

All slopes obtained from the low-energy part of the emission spectra are similar and lie between $144 \mathrm{mV} / \mathrm{eV}$ and $202 \mathrm{mV} / \mathrm{eV}$ with an average of $(178 \pm 22) \mathrm{mV} / \mathrm{eV}$ (see Supplementary Figure 6). The $\Delta V_{\mathrm{nr}}$ vs. $E_{\mathrm{CT}}-\lambda_{\mathrm{s}}$ has a similar slope of either $(-184 \pm 26) \mathrm{mV} / \mathrm{eV}$ or $(-193 \pm 16) \mathrm{mV} / \mathrm{eV}$, depending on the method used to determine $\Delta V_{\mathrm{nr}}$ (for details see Supplementary Table 6). This observation is consistent with a dominant underlying ground-state vibration, similar for all $D: C_{60}$ interfaces, being responsible for both the low energy part of the CT emission spectrum and for the non-radiative recombination. Common high frequency vibrations in organic materials originate from hydrogen-carbon or carbon-carbon type bonds, and are therefore the most likely candidates responsible for the proposed intrinsic decay mechanism ${ }^{31}$.

\section{Nature of the Dominant Skeletal Vibration}

We first chose to evaluate the $\mathrm{C}-\mathrm{H}$ bonds by replacing hydrogen with deuterium, as deuteration has been shown to reduce non-radiative decay of certain CT complexes in solution ${ }^{35}$. The photovoltaic parameters and the EL spectra of $\mathrm{D}: \mathrm{C}_{60}$ devices containing hydrogenated and deuterated rubrene as D however do not differ (see Supplementary Figure 7). Therefore, $\Delta V_{\mathrm{nr}}$ is not affected by deuteration; see Supplementary Table 2 for more details. Having ruled out $\mathrm{C}-\mathrm{H}$ bonds as being the main cause for non-radiative decay, an in-depth analysis of the spectral shape of EL from rubrene: $\mathrm{C}_{60} \mathrm{CT}$ state provides further insight. In this particular case, the relaxation energy $\left(\lambda_{\mathrm{s}}\right)$, originating from the low frequency modes, is exceptionally low which leads to much narrower linewidths allowing the observation of a clearly structured CT emission spectrum. For this material combination we can therefore distinguish the high frequency vibrational modes at low photon-energies as seen in the energetic tail of the EL spectra in Figure 3b. By fitting the data to Equation (4), we extract: $h \bar{v}_{v}=0.16 \mathrm{eV}$ and $\lambda_{\mathrm{s}}=0.09 \mathrm{eV}$. The observed higher frequency vibrational energetic spacing of $h \bar{v}_{v}=0.16 \mathrm{eV}$ $\left(1290 \mathrm{~cm}^{-1}\right)$ is characteristic of carbon-carbon bonds and, accordingly, gives a hint to the characteristic energy range ${ }^{31,18}$ of the vibrations that are responsible for non-radiative recombination in the $\mathrm{D}: \mathrm{C}_{60}$ interfaces studied. 


\section{Impact of Intrinsic Non-Radiative Recombination on the PCE of}

\section{OSCs}

The results presented above suggest an intrinsic and molecular origin of non-radiative recombination in OSCs. Since carbon-carbon vibration are unavoidable for organic materials, this additional voltage loss should be accounted for in the calculation of a theoretical maximum PCE. According to Shockley and Queisser, the upper limit for the PCE an ideal single junction SCs is about $34 \%$ for an optimum bandgap of (1.12-1.38) eV 24,8 , when illuminated with the AM1.5G solar spectrum. Such an ideal SC has an $E Q E_{\mathrm{PV}}$ of $100 \%$ above, and $0 \%$ below the optical gap and non-radiative recombination is absent. If we make the same assumptions but take into account our findings shown in Figure 1b, i.e. intrinsic non-radiative voltage losses obeying the EGL, the theoretical maximum PCE of OSCs reduces to about $25.5 \%$, details on the calculation are presented in Supplementary Note 3. Moreover, the optimal optical gap of the absorber molecules would increase to $(1.45-1.65) \mathrm{eV}$, resulting in a maximum achievable $V_{\mathrm{OC}}$ of $(0.93-1.17) \mathrm{V}$. Assuming non-ideal device parameters such as an overall $E Q E_{\mathrm{PV}}$ of $85 \%{ }^{12}$ and a FF of $80 \%^{3}$, we get a maximum PCE for fullerene containing OSCs of about $19.5 \%$, for a hypothetical electron D with an optical gap of (1.52-1.54) eV and a $V_{O C}$ of $(1.01-1.04) \mathrm{V}$.

\section{Conclusion}

In this work we show that non-radiative voltage losses drastically increase if the energy of the chargetransfer state decreases. This trend is in agreement with the inverted Marcus regime for non-radiative transition rates in organic donor-acceptor systems. Our results can be described by the energy-gap law of non-radiative voltage losses, which is assigned to intramolecular vibrations of the organic semiconductor material itself. We want to emphasize that this proposed loss mechanism is accordingly inherent to organic materials and fundamentally different from the commonly assumed non-radiative loss mechanisms in inorganic semiconductors, which are mainly related to defects, surface recombination, or Auger recombination.

Since we do not see how such non-radiative recombination, mediated by carbon-carbon bonds, can be avoided in future organic molecules the maximal theoretical power conversion efficiency of organic 
solar cells would consequently reduce to about $25.5 \%$ for single junction solar cells and leading to a blue-shifted optimal optical band gap of about (1.45-1.65) eV.

\section{Methods}

Device preparation: The layers of the low-donor-content OSCs are thermally evaporated at ultra-high vacuum (base pressure $<10^{-7}$ mbar) on a glass substrate with a pre-structured ITO contact (Thin Film Devices, USA). For an appropriate hole contact $2 \mathrm{~nm}$ of $\mathrm{MoO}_{3}$ are deposited followed by the "diluted donor" active layer comprising $50 \mathrm{~nm}$ of $\mathrm{C}_{60}$ (CreaPhys $\mathrm{GmbH}$, Germany) doped with 6 mol\% of each D molecule (for more details see Supplementary Table 2). Afterwards, $8 \mathrm{~nm}$ of Bathophenanthroline (BPhen), used as electron contact, is evaporated and finished with $100 \mathrm{~nm}$ of Al. All the organic materials were purified by 2-3 times of sublimation. The device is defined by the geometrical overlap of the bottom and the top contact and equals $6.44 \mathrm{~mm}^{2}$. To avoid exposure to ambient conditions, the organic part of the device was covered by a small glass substrate which is glued on top.

Current-voltage characteristics are measured with a SMU (Keithley 2400, USA) at standard testing conditions (16 S-150 V.3 Solar Light Co., USA) with a mismatch (mismatch $=0.60)$ corrected light intensity. To determine the ideality factor $n_{\text {id }}$ of the SCs, the light intensity is varied in the range of 0.1 suns to 5 suns by using neutral density filters and by changing the distance between sample and light source. In accordance to Reference $10, n_{\text {id }}$ is obtained from the resulting $j_{s c}-V_{O c}$ dependence.

Sensitive $E Q E_{\mathrm{PV}}$ measurements: The light of a quartz halogen lamp $(50 \mathrm{~W})$ is chopped at $140 \mathrm{~Hz}$ and coupled into a monochromator (Newport Cornerstone $2601 / 4 \mathrm{~m}$, USA). The resulting monochromatic light is focused onto the OSC, its current at short-circuit conditions is fed to a current pre-amplifier before it is analysed with a lock-in amplifier (Signal Recovery 7280 DSP, USA). The time constant of the lock-in amplifier was chosen to be $1 \mathrm{~s}$ and the amplification of the pre-amplifier was increased to resolve low photocurrents. The $E Q E_{\mathrm{PV}}$ is determined by dividing the photocurrent of the OSC by the flux of incoming photons, which was obtained with a calibrated silicon (Si) and indium-gallium-arsenide (InGaAs) photodiode.

Electroluminescence measurements were obtained with an Andor SR393i-B spectrometer equipped with a cooled Si and cooled InGaAs detector array (DU420A-BR-DD and DU491A-1.7, UK). The 
spectral response of the setup was calibrated with a reference lamp (Oriel 63355). The emission spectrum of the OSCs was recorded at different injection currents with respect to voltages which were lower than or at least similar to the $V_{O C}$ of the device at 1 sun illumination.

$E Q E_{\mathrm{EL}}$ measurements were conducted by forward biasing the OSCs with an Agilent $4155 \mathrm{C}$ parameter analyser and collecting the emitted radiation by an enhanced G10899-03K InGaAs photodetector from Hamamatsu. The absolute total photon flux determination was performed by placing the OSC at a distance of $18.3 \mathrm{~mm}$ from the photodetector. Knowledge about the spectral distribution of the cell emission, the spectral response of the InGaAs photodetector, and the assumption of a point source emitting uniformly into a half-sphere allows the determination of the absolute EL photon flux from the OSC. Uncertainties in measured $E Q E_{\mathrm{EL}}$ are expected to be governed by the small distance imprecision between the OSC and the photodetector.

\section{Data Availability Statement}

The data that support the plots within this paper and other findings of this study are available from the corresponding authors J.B., K.T., and K.V. upon reasonable request. The data shown in Figure 1 and Figure 3a is listed in Supplementary Tables 3, 4, and 5.

\section{References}

1. Park, SH. et al., Bulk heterojunction solar cells with internal quantum efficiency approaching $100 \%$. Nat Photonics 3 (5), 297-303 (2009).

2. Liu, Y. et al., Aggregation and morphology control enables multiple cases of high-efficiency polymer solar cells. Nat Commun 5, 5293 (2014).

3. Guo, X. et al., Polymer solar cells with enhanced fill factors. Nat Photonics 7 (10), 825-833 (2013).

4. Zhao, J. et al., Efficient organic solar cells processed from hydrocarbon solvents. Nat Energy 1, 15027 (2016). 
5. Green, MA., Radiative Efficiency of State-of-the-Art Photovoltaic Cells. Prog Photovoltaics 20, 472476 (2012).

6. Tvingstedt, K. et al., Radiative efficiency of lead iodide based perovskite solar cells. Sci Rep 4, $6071(2014)$.

7. Würfel, P., Physics of Solar Cells: From Basic Principles to Advanced Concepts, 2nd ed. (WileyVCH, Weinheim, 2009).

8. Queisser, HJ., Detailed balance limit for solar cell efficiency. Mater Sci Eng B 159-160, 322-328 (2009).

9. Vandewal, K., Tvingstedt, K., Gadisa, A., Inganäs, O. \& Manca, JV., Relating the open-circuit voltage to interface molecular properties of donor:acceptor bulk heterojunction solar cells. Phys Rev B 81 (12), 125204 (2010).

10. Tvingstedt, K. \& Deibel, C., Temperature Dependence of Ideality Factors in Organic Solar Cells and the Relation to Radiative Efficiency. Adv Energy Mater (1502230), 1-13 (2016).

11. Ran, NA. et al., Harvesting the Full Potential of Photons with Organic Solar Cells. Adv Mater 28 (7), 1482-1488 (2016).

12. Liu, J. et al., Fast charge separation in a non-fullerene organic solar cell with a small driving force. Nat Energy 1 (9), 16089 (2016).

13. Vandewal, K. et al., Quantification of Quantum Efficiency and Energy Losses in Low Bandgap Polymer:Fullerene Solar Cells with High Open-Circuit Voltage. Adv Funct Mater 22, 3480-3490 (2012).

14. Wang, C. et al., Low Band Gap Polymer Solar Cells With Minimal Voltage Losses. Adv Energy Mater 6 (18), 1600148 (2016).

15. Vandewal, K. et al., Increased Open-Circuit Voltage of Organic Solar Cells by Reduced DonorAcceptor Interface Area. Adv Mater 26 (23), 3839-3843 (2014).

16. Rau, U., Reciprocity relation between photovoltaic quantum efficiency and electroluminescent emission of solar cells. Phys Rev B 76, 1-8 (2007). 
17. Yao, J. et al., Quantifying Losses in Open-Circuit Voltage in Solution-Processable Solar Cells. Phys Rev Appl 4 (1), 014020 (2015).

18. Gould, IR. et al., Radiative and nonradiative electron transfer in contact radical-ion pairs. Chem Phys 176 (2-3), 439-456 (1993).

19. Englman, R. \& Jortner, J., The energy gap law for radiationless transitions in large molecules. Mol Phys 18 (2), 145-164 (1970).

20. Kalyanasundaram, K. \& Nazeeruddin, MK., Tuning of the CT excited state and validity of the energy gap law in mixed ligand complexes of Ru(II) containing 4,4'-dicarboxy-2,2'-bipyridine. Chem Phys Lett 193 (4), 292-297 (1992).

21. Siebrand, W., Radiationless Transitions in Polyatomic Molecules. II. Triplet-Ground-State Transitions in Aromatic Hydrocarbons. J Chem Phys 47 (7), 2411 (1967).

22. Gould, IR. \& Farid, S., Radiationless decay in exciplexes with variable charge transfer. J Phys Chem B 111 (24), 6782-6787 (2007).

23. Mataga, N. et al., First Unequivocal Observation of the Whole Bell-Shaped Energy Gap Law in Intramolecular Charge Separation from S2 Excited State of Directly Linked Porphyrin-Imide Dyads and Its Solvent-Polarity Dependencies. J Am Chem Soc 123 (49), 12422-12423 (2001).

24. Shockley, W. \& Queisser, HJ., Detailed balance limit of efficiency of p-n junction solar cells. J Appl Phys 32 (3), 510-519 (1961).

25. Vandewal, K., Tvingstedt, K., Gadisa, A., Inganäs, O. \& Manca, JV., On the origin of the opencircuit voltage of polymer-fullerene solar cells. Nat Mater 8 (11), 904-909 (2009).

26. Piersimoni, F. et al., Influence of fullerene ordering on the energy of the charge-transfer state and open-circuit voltage in polymer:fullerene solar cells. J Phys Chem C 115 (21), 10873-10880 (2011).

27. Vandewal, K. et al., Varying polymer crystallinity in nanofiber poly(3-alkylthiophene): PCBM solar cells: Influence on charge-transfer state energy and open-circuit voltage. Appl Phys Lett 95 (12), 21-24 (2009).

28. Tietze, ML. et al., Correlation of open-circuit voltage and energy levels in zinc-phthalocyanine:C60 bulk heterojunction solar cells with varied mixing ratio. Phys Rev B 88 (8), 1-10 (2013). 
29. Zhang, M., Wang, H., Tian, H., Geng, Y. \& Tang, CW., Bulk heterojunction photovoltaic cells with low donor concentration. Adv Mater 23 (42), 4960-4964 (2011).

30. Nitzan, A., Mukamel, S. \& Jortner, J., Energy gap law for vibrational relaxation of a molecule in a dense medium. J Chem Phys 63 (1), 200-207 (1975).

31. Wilson, JS. et al., The energy gap law for triplet states in Pt-containing conjugated polymers and monomers. J Am Cheml Soc 123 (38), 9412-9417 (2001).

32. Marcus, RA., Electron transfer reactions in chemistry. Theory and experiment. Rev Mod Phys 65 (3), 599-610 (1993).

33. Gould, IR., Farid, S. \& Young, RH., Relationship Between Exciplex Fluorescence and ElectronTransfer in Radical Ion-Pairs. J Photoch Photobio A 65 (1-2), 133-147 (1992).

34. Vandewal, K. et al., Charge-Transfer Absorption Tails of Photovoltaic Donor:C60 Blends Provide Insight into Thermally Activated Vibrations and Polaron Relaxation. J Am Chem Soc (139), 16991704 (2017).

35. Kulinowski, K., Gould, IR., Ferris, NS. \& Myers, AB., Spectroscopic, Kinetic, and Thermodynamic Deuterium Isotope Effects in the HexarnethylbenzenelTetracyanoethylene Charge-Transfer Complex. J Phys Chem 99 (50), 17715-17723 (1995).

36. Fang, L. et al., Thermally Stable Fluorine-Containing Low-Energy-Gap Organic Dyes with Low Voltage Losses for Organic Solar Cells. Synthetic Met 222, 232-239 (2016).

37. Moench, T. et al., Influence of Meso and Nanoscale Structure on the Properties of Highly Efficient Small Molecule Solar Cells. Adv Energy Mater 6 (4), 1-10 (2016).

38. Koerner, C. et al., Probing the effect of substrate heating during deposition of DCV4T:C60 blend layers for organic solar cells. Org Electron 13 (4), 623-631 (2012).

39. Fitzner, R. et al., Correlation of pi-conjugated oligomer structure with film morphology and organic solar cell performance. J Am Chem Soc 134 (27), 11064-11067 (2012).

40. Wang, E. et al., Conformational disorder enhances solubility and photovoltaic performance of a thiophene-quinoxaline copolymer. Adv Energy Mater 3 (6), 806-814 (2013). 
41. Vandewal, K. et al., The relation between open-circuit voltage and the onset of photocurrent generation by charge-transfer absorption in polymer:fullerene bulk heterojunction solar cells. Adv Funct Mater 18, 2064-2070 (2008).

42. Hoke, ET. et al., Recombination in polymer:Fullerene solar cells with open-circuit voltages approaching and exceeding 1.0 V. Adv Energy Mater 3 (2), 220-230 (2013).

43. Tang, Z. et al., A New Fullerene-Free Bulk-Heterojunction System for Efficient High-Voltage and High-Fill Factor Solution-Processed Organic Photovoltaics. Adv Mater 27 (11), 1900-1907 (2015).

\section{Corresponding Authors}

Correspondence and request for materials should be addressed to J.B., K.T., or K.V.

\section{Acknowledgements}

This work was supported by the German Federal Ministry for Education and Research (BMBF) through the InnoProfille project "Organische p-i-n Bauelemente 2.2". K.T. acknowledges the People Programme (Marie Curie Actions) of the European Union's Seventh Framework Programme FP7 under the REA grant agreement PIEF-GA-2012-327199. F.P. and D.N. acknowledge funding by the German Research Foundation (DFG) via the SFB 951 "HIOS". The work of Georgia Tech was supported by the Department of the Navy, Office of Naval Research Award No. N00014-14-1-0580 (CAOP MURI), and through a State-Sponsored Scholarship for Graduate Students to Y.F. from the China Scholarship Council. M.T. thanks the Christ Church Oxford for financial support with a Junior Research Fellowship. M.K.R. acknowledges the UK Engineering and Physical Science Research Council (EPSRC) through grant EP/L026066/1.

Additionally, we thank for the supply of the donor molecules: Prof. Bäuerle from University of Ulm for $\mathrm{DH} 4 \mathrm{~T}, \mathrm{DH} 6 \mathrm{~T}$ and several $\mathrm{DCV}_{2}-n \mathrm{~T}$, Markus Hummert for P4-Ph4-DIP and BP-Bodipy, and Beatrice Beyer for $\mathrm{ZnF}_{4} \mathrm{Pc}$. Furthermore, we acknowledge Felix Holzmueller, Christian Koerner, Matthias Saalfrank, and Rico Meerheim for providing OSC devices for this study. 


\section{Author Contribution}

J.B., K.T., D.S., and K.V. designed the experiments, prepared photovoltaic devices and optimized their processing parameters for photovoltaic performance. J.B. measured the sensitive EQE $E_{\mathrm{PV}}$ spectra, K.T. measured the $E Q E_{\mathrm{EL}}$, and F.P. measured the corresponding electroluminescence spectra. D.S., J.B., and S.U. performed the standard characterization of the solar cells. M.T., Y.F., and O.Z. synthesized donor molecules for low $E_{\text {Ст }}$ systems, important for this study. K.A.M. synthesized deuterated rubrene. D.N., S.B., S.R.M., M.K.R., and C.J.D. supervised their team members involved in the project. K.V. supervised the overall project. All authors contributed to analysis and writing.

\section{Competing Financial Interests}

The authors declare no competing financial interests.

\section{Figure Captions}

Figure 1 | $V_{\mathrm{OC}}$ and Non-Radiative Voltage Losses as Function of $E_{\mathrm{CT}}$ a $V_{\mathrm{OC}}$ at 1 sun illumination of D:fullerene OSCs consisting of different D molecules, as a function of $E_{\mathrm{CT}}$. The overall voltage losses, $\Delta V_{\mathrm{OC}}=E_{\mathrm{CT}} / q-V_{\mathrm{OC}}$, decrease with increasing $E_{\mathrm{CT}}$. The red line shows the ideal $V_{\mathrm{OC}}$, i.e. at $\mathrm{SQ}$ assumptions, as a function of $E_{\mathrm{CT}}$. $\mathbf{b} \Delta V_{\mathrm{nr}}$ as a function of the $E_{\mathrm{CT}}$. Filled symbols show data calculated from $E Q E_{\mathrm{PV}}$ and unfilled symbols correspond to data measured by $E Q E_{\mathrm{EL}}$. The red dashed line indicates an empirical lower limit of $\Delta V_{\mathrm{nr}}$ as a function of $E_{\mathrm{CT}}$.

Circles show BHJ OSCs whereas squares represent PHJ devices. Black symbols show a specific series of devices for which SM Ds are diluted at 6 mol\% in $\mathrm{C}_{60}$ molecules (see Supplementary Table 3), blue symbols indicate SM Ds at usual D:A concentration (details of selected OSCs were published in ${ }^{36,37,38,39}$, see Supplementary Table 4), and green symbols represent devices comprising polymer Ds mixed with fullerenes ${ }^{40,9,25,27,41,42,13}$ for which the sensitively measured EQE spectra were reanalysed for this work (see Supplementary Table 5). Crosses indicate recently published OSCs for which data was available $43,14,12,11$ (see Supplementary Table 5). 
Figure 2 | Recombination Mechanism from the Excited CT state to the Ground State. The potential energies of the ground state and the CT state at D-A interface as a function of the reaction coordinate are parabolic. The sublevels indicate vibrational excited states and their wave-functions are indicated by the grey areas. Non-radiative $\left(k_{\mathrm{nr}}\right)$ and radiative $\left(k_{\mathrm{r}}\right)$ transitions from the excited state $\left(\mathrm{D}^{+} / \mathrm{A}^{-}\right)$, the $\mathrm{CT}$ state, to the ground state $(\mathrm{D} / \mathrm{A})$ are illustrated $\mathbf{a}$ for a high $E_{\mathrm{CT}}$ and $\mathbf{b}$ for a low $E_{\mathrm{CT}}$. A non-radiative transition takes place mainly from the relaxed CT state $(i=0)$ to the vibrationally excited ground state $(j=n)$ and thermalizes afterwards. The transition probability is sketched by the green area being the wave-function overlap of both states. For emission, the electron directly transfers to the relaxed ground state or a low vibrational mode of the ground state (red arrows).

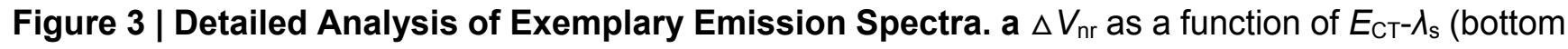
$x$-axis) for a range of different $\mathrm{D}: \mathrm{C}_{60}$ combinations. Furthermore, the emission spectrum of 4 selected OSCs is shown as function of -hv shifted by the $E_{\mathrm{CT}-} \lambda_{\mathrm{s}}$, which equals the peak maximum of the emission (top $x$-axis). The scale of non-radiative recombination and the emission are chosen to be equivalent. Both radiative and non-radiative recombination depend on the $F C$ factor, see Equation (2) and Equation (4), therefore, the slope of both is similar. For details see the main text. The error bar of $\Delta V_{\mathrm{nr}}$ is obtained by systematic variation of the fit range of the SEQE absorption tail (standard deviation of 25 fits). b Normalized reduced EL spectra and reduced $E Q E_{\mathrm{PV}}$ of the $\mathrm{C}_{60}$ :rubrene (17:1) CT state, measured at $0.93 \mathrm{~V}$ and $1.10 \mathrm{~V}$. Emission peaks at lower photon energies, indicated by black, vertical lines, can be attributed to transitions from the lowest energy CT state to high frequency vibrational modes of the ground state (i.e. $j=\{0,1,2\})$. The crossing point of both spectra determines $E_{\mathrm{CT}}$. 
- Fit of BHJ (low donor SM)

- SQ limit for $V_{O C}$

$\mathrm{E}_{\mathrm{CT}} / \mathrm{q}-0.5 \mathrm{~V}$

$\cdots \mathrm{E}_{\mathrm{CT}} / \mathrm{q}-0.6 \mathrm{~V}$

-.- $E_{C T} / q-0.7 V$

- $\operatorname{BHJ}(\mathrm{SM})$

- $\mathrm{PHJ}(\mathrm{SM})$

- BHJ (polymer)

$\times \quad \mathrm{BHJ}$ (polymer; literature)
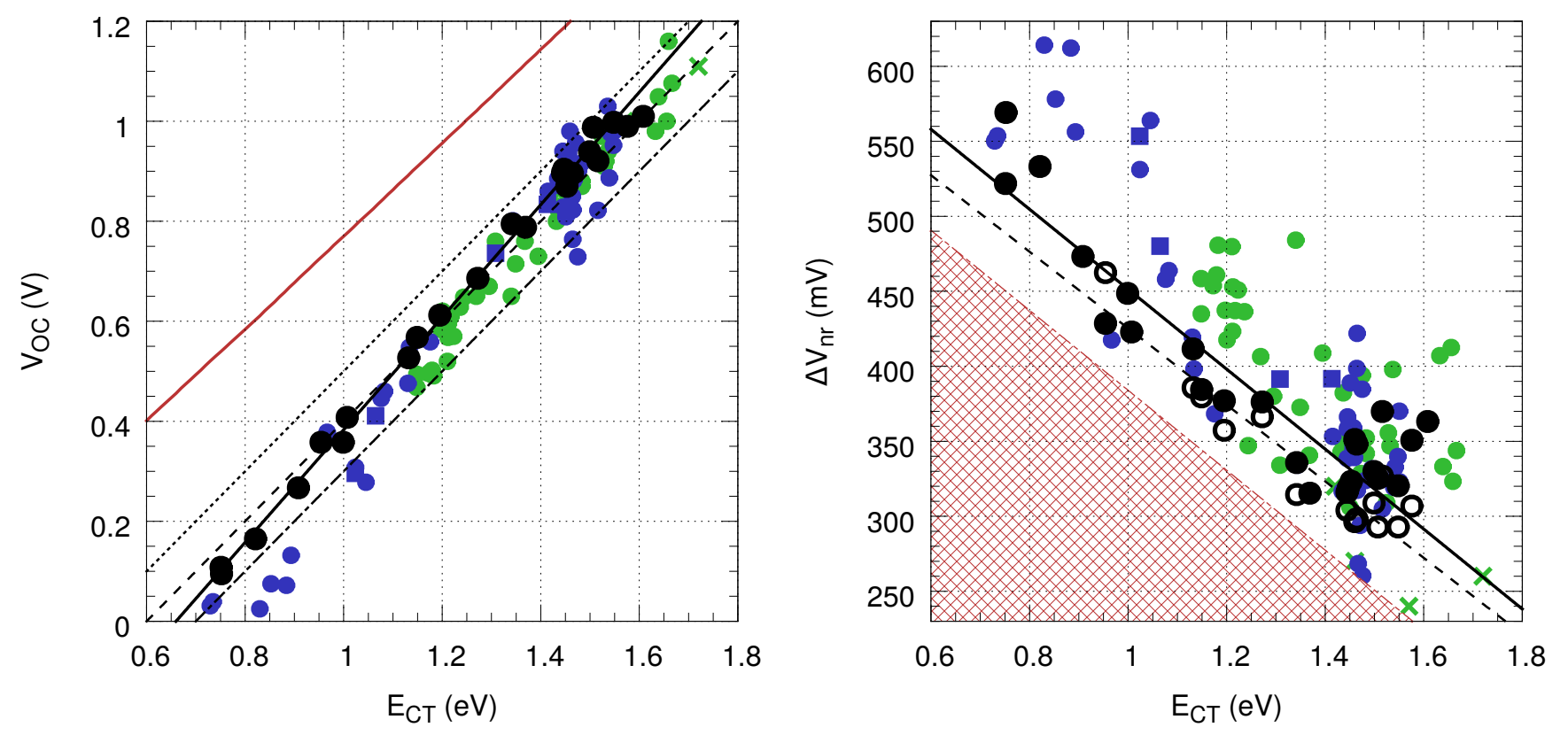

- $\mathrm{BHJ}$ (low donor $\mathrm{SM}$; from $\mathrm{EQE}_{\mathrm{PV}}$ )

- Fit of $\Delta \mathrm{V}_{\mathrm{nr}}$ (from $\mathrm{EQE}_{\mathrm{PV}}$ data)

- BHJ (low donor $S M$; from $\mathrm{EQE}_{\mathrm{EL}}$ )

- - - Fit of $\Delta \mathrm{V}_{\mathrm{nr}}$ (from $\mathrm{EQE}_{\mathrm{EL}}$ data)

----- lower limit of $\Delta \mathrm{V}_{\mathrm{nr}}\left(\mathrm{E}_{\mathrm{CT}}\right)$ 
a | High $E_{\mathrm{CT}}$

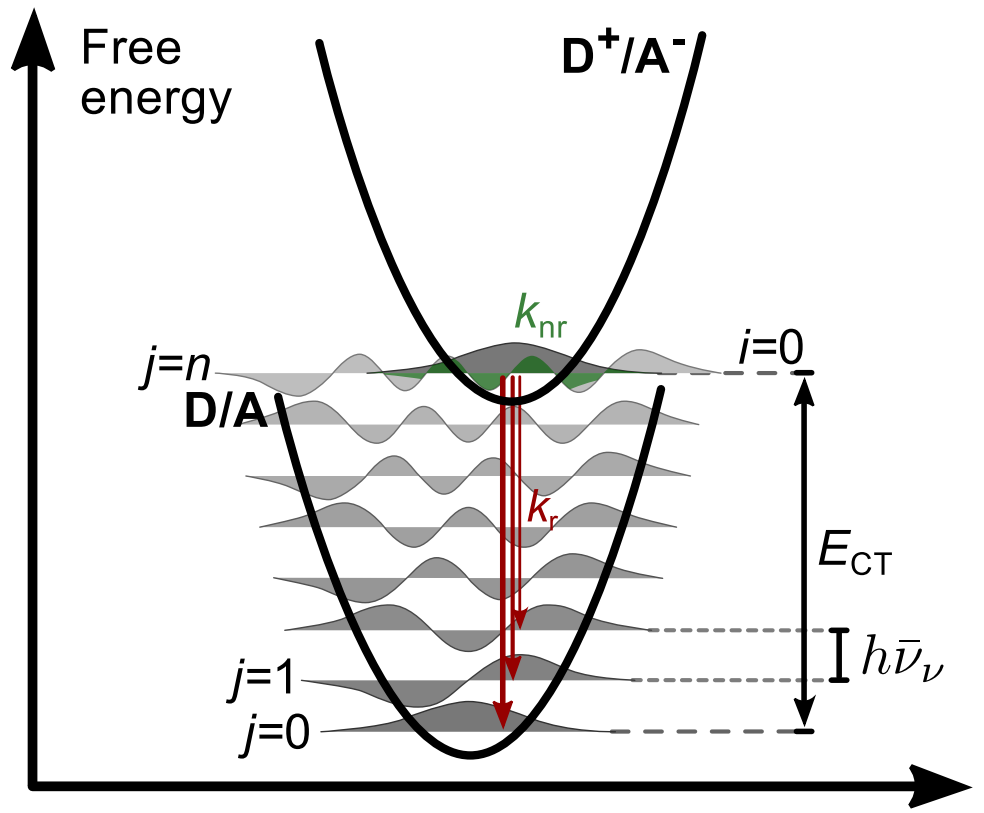

Reaction coordinate (intra-molecular) b | Low $E_{\mathrm{CT}}$
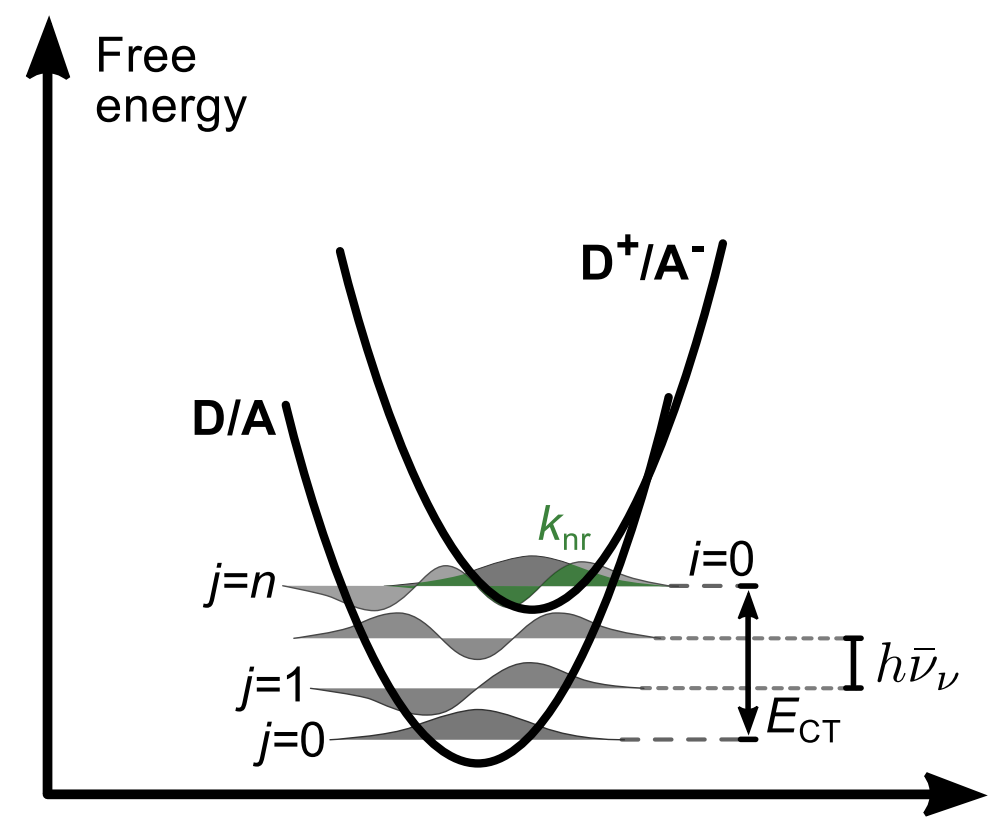

Reaction coordinate (intra-molecular) 
